\title{
Bowel Movement Patterns and Constipation Prevalence in School Children of South Jordan
}

\author{
Eyad Altamimi ${ }^{a}$, c, Mohammad Al-Safadi ${ }^{b}$
}

\begin{abstract}
Background: Pediatric constipation is a common problem worldwide. Diet is one of the major determinants of bowel movement patterns, including the presence of constipation. Little is known about bowel movement pattern in Jordanian children. Parents might not recognize constipation in school children, which could lead to delayed treatment and increase rate of complication. This study aimed to investigate bowel motion pattern and constipation prevalence in Jordanian children using self-reporting.
\end{abstract}

Methods: Six schools in Alkarak governorate were selected randomly. Students of grades 6 - 8 were asked to fill the Arabic version of Questionnaire on Pediatric Gastrointestinal Symptoms-Rome III (QPGS-RIII) questionnaire. Children were diagnosed with constipation if two or more criteria on the QPGS-RIII were met. Fisher's exact test was used to analyze categorical data, while Student's $t$-test was used for continuous data.

Results: Of 429 questionnaires administered, 413 (96.3\%) were completed (males: 50.8\%; mean age: 12.7 years old (range: 11 - 16 years old)). A statistically significant sex difference in the presence of hard, bulky bowel movements and fecal incontinence was observed ( $\mathrm{P}=$ $0.000,0.041$ and 0.002 , respectively). In our cohort, 110 (26.6\%) children met Rome III criteria for constipation, which was not statistically significant by sex (59 (28.1\%) versus 51 (25.1\%), male versus female participants, respectively; $\mathrm{P}=0.491$ ).

Conclusion: Most Jordanian school children reported a daily soft bowel movement without pain or retention. Constipation affects approximately $25 \%$ of Jordanian school children between 11 and 16 years old.

Keywords: Diet; Gastrointestinal symptoms; Pediatric; Bowel habits

Manuscript submitted September 23, 2018, accepted October 8, 2018

aPediatric Department, Faculty of Medicine, Jordan University of Science and Technology, PO Box 3030, Irbid 22110, Jordan

${ }^{b}$ World Health Organization, Boston, MA, USA

${ }^{c}$ Corresponding Author: Eyad Altamimi, Pediatric Department, Faculty of Medicine, Jordan University of Science and Technology, PO Box 3030, Irbid 22110, Jordan. Email: emaltamimi@just.edu.jo

doi: https://doi.org/10.14740/ijcp318

\section{Introduction}

Pediatric constipation is a common problem worldwide [1-4]. Previous studies have shown that mean bowel frequency is reduced significantly in children with a positive family history (i.e. parents and/or siblings) of constipation [5]. Adults with constipation consider constipation in their children to be a familial issue and will not report symptoms unless asked, which leads to delayed diagnosis. Failure to recognize constipation in school children may lead to delayed treatment and has been associated with complications (e.g. fecal incontinence) and poorer long-term outcomes (e.g. persistence of symptoms and continuous need for laxatives) [6].

Normal bowel movement patterns vary across individuals. Bowel movement patterns refer to the frequency, consistency and size of passed stool [7]. Bowel movements become less frequent with age, and patterns stabilize by 4 years of age [5]. Environmental factors, including diet, social background and parental behavior, as well as genetics, are believed to influence bowel movement patterns [5]. A physician needs to understand the normal bowel movement pattern of a patient in order to diagnose whether gastrointestinal symptoms deviate from normal.

Diet is one of the major determinants of bowel movement patterns [8]. South Asian children consume rice-based diets with higher fiber content than diets of Western children, who consume less fiber and have more frequent bowel movements [8]. The diet of Jordanian children is a mixture of Eastern and Western diets. There are no studies on the impact of the Jordanian diet on pediatric constipation. Furthermore, data from either Eastern or Western countries may not be generalizable due to unmatched conditions that affect bowel movement patterns. Therefore, in this project, we aimed to study bowel movement patterns and prevalence of constipation among Jordanian school children.

\section{Materials and Methods}

A cross-sectional school-based study was conducted in Alkarak Governorate of Jordan, between October 2012 and April 2013. Participating schools were randomly selected from the whole geographical area. The research team contacted the schools in advance, to obtain the mandatory permissions. Six schools participated. In each school, classes from academic years (grades) $6-8$ were selected. All students attending the class on the day of the survey were included. Children who refused to partici- 
Table 1. Patients' Characteristics

\begin{tabular}{ll}
\hline Male, $\mathrm{n}(\%)$ & $210(50.8)$ \\
Female, $\mathrm{n}(\%)$ & $203(49.2)$ \\
Age, mean (range in years) & $12.7(11-16)$ \\
\hline
\end{tabular}

pate were excluded.

Data on bowel movement characteristics were gathered using the using the Arabic self-reported Questionnaire on Pediatric Gastrointestinal Symptoms-Rome III Version (QPGSRIII). The original questionnaire was translated and validated into Arabic language in cooperation with the Rome Foundation. Rome foundation granted the researchers the permission to use the questionnaires.

On the day of the survey, questionnaires were distributed in the classrooms; students were asked to consider themselves in exam; research team was available to explain the process and answer any inquiries; no time limit was given to fill the questionnaires; and confidentiality and privacy were insured.

No demographic information was collected except age, grade and gender.

Children were considered constipated if they met QPGSRIII criteria for constipation [9], which includes at least two of the following: 1) two or fewer defecations per week; 2) at least one episode of fecal incontinence per week; 3 ) a history of retentive posturing or excessive volitional stool retention; 4) a history of painful or hard bowel movements; 5) the presence of large fecal mass in the rectum; or 6) a history of large-diameter stools that may obstruct the toilet.

\section{Statistical analysis}

SPSS Statistical Package Version 17 (IBM, Armonk, NY, USA) was used. Results are expressed as numbers and percentages. Fisher's exact test was used to analyze categorical data, while Student's $t$-test was used for continuous data. $\mathrm{P}<$ 0.05 was considered significant.

This study was granted ethical approval by the Ethics Committee of Medical School, Mutah University. The data collected were anonymous with no identifiers and kept confidential.

\section{Results}

A total of 429 questionnaires were distributed. Of 413 (96.3\%) completed questionnaires, $50.8 \%$ were completed by male participants. The mean age of participants was 12.7 years (range: 11 - 16 years) (Table 1). A total of 252 (61\%) participants reported having at least one bowel movement per day, whereas $123(29.8 \%)$ reported having two or less bowel movements per week. In addition, $69(16.7 \%)$ described their bowel movement as hard or very hard, $85(20.6 \%)$ reported painful defecation, $115(27.8 \%)$ reported stool retention at least once per week, $69(16.7 \%)$ reported fecal incontinence at least once per week and $84(20.3 \%)$ reported bulky stool. Male participants reported hard bulky bowel movements and incontinence more frequently than female participants, which was statistically significant $(\mathrm{P}=0.000,0.041$ and 0.002 , respectively). Details of bowel movement characteristics stratified by sex are reported in Table 2.

In our cohort, 110 (26.6\%) children met Rome III criteria for constipation. There was no significant sex difference in constipation (59 $(28.1 \%)$ versus $51(25.1 \%)$, male versus female participants, respectively; $\mathrm{P}=0.491$ ).

\section{Discussion}

The present study evaluated the bowel movement patterns of Jordanian school children between the ages of 11 and 16 years old. Most Jordanian school children reported a daily soft bowel movement without pain or retention. Constipation affects approximately $25 \%$ of Jordanian school children between 11 and 16 years old.

In our cohort, 290 (70.2\%) participants reported having a bowel movement three or more times per week, which was less frequent than children from South Asia $[8,10,11]$.

This finding may be due to differences in diet; while the South Asian diet is high in fiber, the westernization of the Jordanian diet limits fiber intake.

Previous studies did not find significant sex differences in bowel frequency $[5,8,10]$. In contrast, our study revealed that female Jordanian school children report a statistically significant higher frequency of bowel movements (two to three times per day) than male children $(\mathrm{P}=0.002)$. However, our findings may reflect over- or under-reporting by responders rather than a true sex difference.

In our study, large-diameter stools, withholding posture, and harder stools were more common in male participants, which are consistent with previous reports from Sri Lanka and the USA $[8,12]$. Compared to Sri Lankan children, male participants in our cohort also reported pain with defecation more frequently than female participants [8]. A previous study from Italy reported a positive association between decreased defecation frequency and presence of hard stools and painful defecation [5], which is consistent with our findings.

Parents do not always recognize constipation in school children. Failure of parents and doctors to recognize constipation can lead to chronic symptoms, development of complications and high rates of treatment failure. Fecal incontinence is known to be related to prolonged untreated constipation [13]. In our cohort, encopresis was reported more often by male than female participants, which is consistent with previous studies $[14,15]$.

In our cohort, $20.1 \%$ of children reported having fecal incontinence at least once per month, which is a higher percentage than previously reported among school children $[14,15]$. If fecal matter streaks in underpants, which might reflect a hygiene issue or poor toilet paper use, are excluded, then the rate of fecal incontinence drops by $50 \%$ in our cohort. Although a $10 \%$ rate of fecal incontinence is still high, this finding is somewhat consistent with a previous study in Brazilian children (14\% versus $19 \%$ between non-constipated and consti- 
Table 2. Bowel Habits' Features According to Gender in School Children

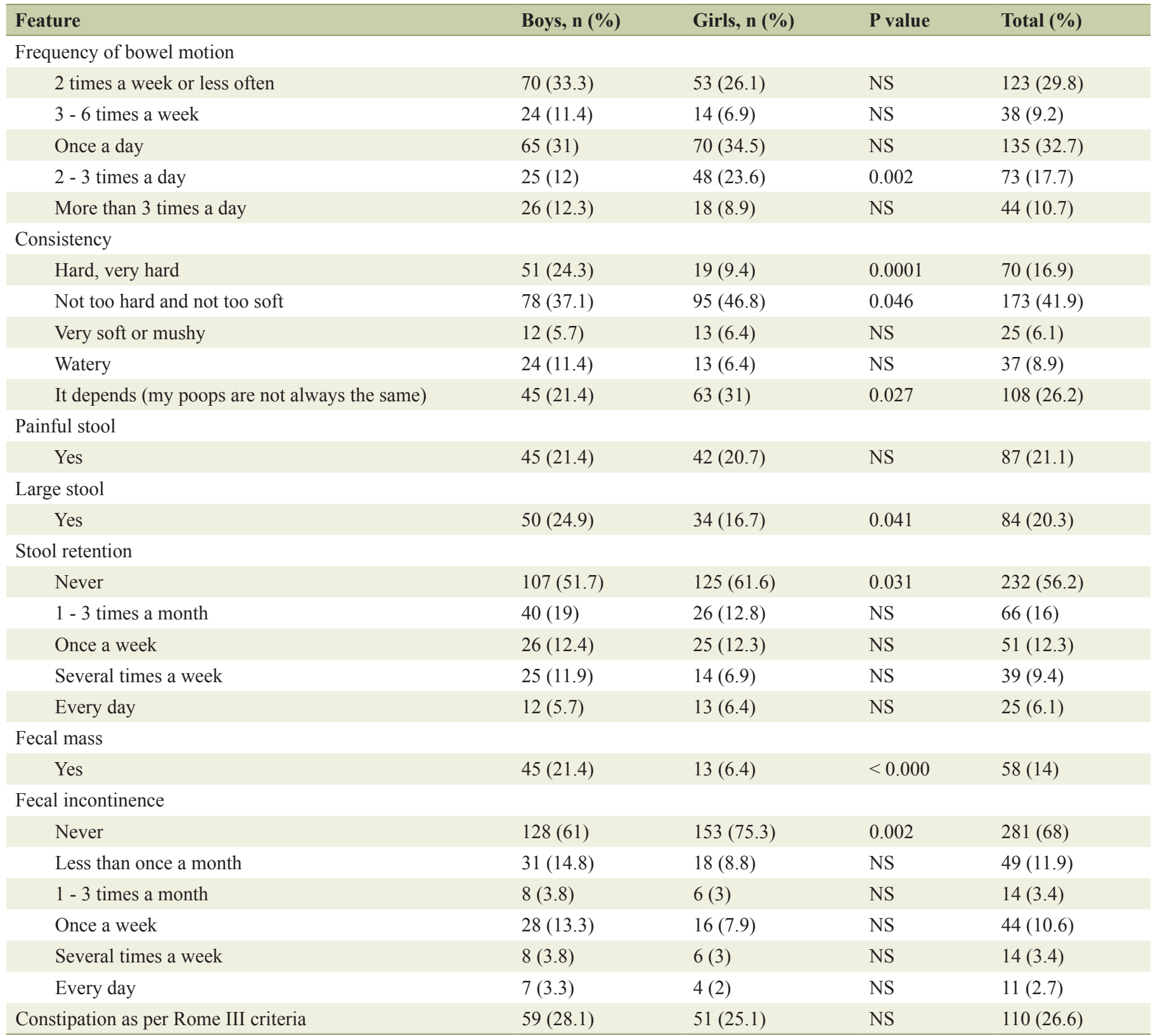

NS: not significant.

pated children, respectively) [16].

In our cohort, $110(26.6 \%)$ participants met the criteria for constipation, which is also consistent with a previous Brazilian study [16]. In that study, a questionnaire inquiring about difficulties during defecation was administered to children between 8 and 10 years of age. The study found that $28 \%$ of children were constipated according to the study's definition [16]. Another study from the USA reported that self-reported constipation occurred in an average of $18 \%$ of children (range: 7-39\%).

Still our study suffers some limitations. Bowel movements are a sensitive issue, which many children feel shy or uncom- fortable discussing. Although this study reports valuable data about bowel movements in Jordanian school children, recall bias and under- or over-reporting of symptoms cannot be ignored. This study, although not designed for at first, lacks clinical evaluation of the study subjects.

Factors affecting the bowel movements were not addressed in this study. Further studies are needed to study the socioeconomic and psychological factors that affect bowel movements in this population.

In conclusion, this study provides important data about bowel movement patterns and constipation in Jordanian school children. Most Jordanian school children have a daily soft 
bowel movement without pain or retention. Constipation affects approximately $25 \%$ of Jordanian school children between the ages of 11 and 16 years old.

\section{Presentation}

This work was presented at its abstract form at the ESPGHAN meeting, 2015 at Amsterdam, The Netherlands.

\section{Financial Support}

None.

\section{References}

1. Pashankar DS. Childhood constipation: evaluation and management. Clin Colon Rectal Surg. 2005;18(2):120127.

2. Agarwal J. Chronic constipation. Indian J Pediatr. 2013;80(12):1021-1025.

3. Caplan A, Walker L, Rasquin A. Validation of the pediatric Rome II criteria for functional gastrointestinal disorders using the questionnaire on pediatric gastrointestinal symptoms. J Pediatr Gastroenterol Nutr. 2005;41(3):305316.

4. van den Berg MM, Benninga MA, Di Lorenzo C. Epidemiology of childhood constipation: a systematic review. Am J Gastroenterol. 2006;101(10):2401-2409.

5. Corazziari E, Staiano A, Miele E, Greco L, Italian Society of Pediatric Gastroenterology, Hepatology, and Nutrition. Bowel frequency and defecatory patterns in children: a prospective nationwide survey. Clin Gastroenterol Hepatol. 2005;3(11):1101-1106.

6. Loening-Baucke V. Constipation in early childhood: pa- tient characteristics, treatment, and longterm follow up. Gut. 1993;34(10):1400-1404.

7. Weaver LT. Bowel habit from birth to old age. J Pediatr Gastroenterol Nutr. 1988;7(5):637-640.

8. Devanarayana NM, Rajindrajith S. Bowel habits and behaviors related to defecation in 10- to 16-year-olds: impact of socioeconomic characteristics and emotional stress. J Pediatr Gastroenterol Nutr. 2011;52(5):569-573.

9. Rasquin A, Di Lorenzo C, Forbes D, Guiraldes E, Hyams JS, Staiano A, Walker LS. Childhood functional gastrointestinal disorders: child/adolescent. Gastroenterology. 2006;130(5):1527-1537.

10. Osatakul S, Yossuk P, Mo-suwan L. Bowel habits of normal Thai children. J Pediatr Gastroenterol Nutr. 1995;20(3):339-342.

11. Myo K, Thein Win N, Kyaw-Hla S, Thein Thein M, Bolin TD. A prospective study on defecation frequency, stool weight, and consistency. Arch Dis Child. 1994;71(4):311313; discussion 313-314.

12. Wald ER, Di Lorenzo C, Cipriani L, Colborn DK, Burgers R, Wald A. Bowel habits and toilet training in a diverse population of children. J Pediatr Gastroenterol Nutr. 2009;48(3):294-298.

13. Rajindrajith S, Devanarayana NM, Benninga MA. Review article: faecal incontinence in children: epidemiology, pathophysiology, clinical evaluation and management. Aliment Pharmacol Ther. 2013;37(1):37-48.

14. Rajindrajith S, Devanarayana NM, Mettananda S, et al. Constipation and functional faecal retention in a group of school children in a district in Sri Lanka. Sri J Child Health. 2009;38:60-64.

15. van der Wal MF, Benninga MA, Hirasing RA. The prevalence of encopresis in a multicultural population. J Pediatr Gastroenterol Nutr. 2005;40(3):345-348.

16. de Araujo Sant'Anna AM, Calcado AC. Constipation in school-aged children at public schools in Rio de Janeiro, Brazil. J Pediatr Gastroenterol Nutr. 1999;29(2):190-193. 